\title{
Machine Learning Driven Human Skill Transferring for Control of Anthropomorphic Manipulators
}

\author{
Hang $\mathrm{Su}^{1}$, Wen $\mathrm{Qi}^{1}$, Hongbo $\mathrm{Gao}^{2}$, Yingbai $\mathrm{Hu}^{3}$, Yan $\mathrm{Shi}^{4}$, Giancarlo Ferrigno ${ }^{1}$ and Elena De Momi ${ }^{1}$
}

\begin{abstract}
The kinematic mapping between human arm motions and anthropomorphic manipulators are introduced to transfer human skill and to accomplish human-like behavior for control of anthropomorphic manipulators. The availability of big data and machine learning facilitates imitation learning for anthropomorphic robot control. In this paper, a machine learning-driven human skill transferring for control of anthropomorphic manipulators is proposed. The proposed deep convolutional neural network (DCNN) model utilizes a swivel motion reconstruction approach to imitate human-like behavior for fast and efficient learning. Finally, the trained neural network is translated to manage the redundancy optimization control of anthropomorphic robot manipulators. This approach also holds for other redundant robots with anthropomorphic kinematic structure.
\end{abstract}

\section{INTRODUCTION}

Human-like practice imitation has attracted increasing research attention in anthropomorphic robotics control through the prior decades. It has been demonstrated that the humanlike motion control of anthropomorphic manipulators is capable of enhancing the quality of Human-Robot Interaction (HRI) [1] prominently in multiple areas, like industry and bio-medical engineering purposes [2]-[4]. Especially for the anthropomorphic serial robot with human-like mechanical structures, for example, YuMi (ABB, Zurich, Switzerland), LWR4+ (KUKA, Augsburg, Germany), and Justin robot (Institute of Robotics and Mechatronics, Wessling, Germany). It is effective to achieve more social, cognitive, and reasonable in human-like behavior.

There are many investigations into the manipulators in human-like motion. For example, an autonomous adaptation human-like control for reaching the target of hands-on

\footnotetext{
*This work was supported by the European Commission Horizon 2020 research and innovation program, under the project SMARTsurg, grant agreement No. 732515, and in part by "the Fundamental Research Funds for the Central Universities", "the Science and Technology Innovation Planning Project of Ministry of Education of China", "NVIDIA NVAIL program", "the National Natural Science Foundation of China under Grant No. U1804161", and Key Laboratory of Advanced Perception and Intelligent Control of High-end Equipment of Ministry of Education (Anhui Polytechnic University, Wuhu, China, 241000) under Grant Nos. GDSC202001 and GDSC202007. Corresponding author: Hongbo Gao

${ }^{1}$ Hang Su, Wen Qi, Giancarlo Ferrigno and Elena De Momi are with the Department of Electronics, Information and Bioengineering, Politecnico di Milano, 20133, Milan, Italy. hang.su, wen.qi, giancarlo.ferrigno, elena.demomiepolimi.it.

${ }^{2}$ Hongbo Gao is with the Department of Automation, University of Science and technology of China, Hefei, China. ghb48@126.com.

${ }^{3}$ Yingbai Hu is with the Department of Informatics, Technical University of Munich, Munich, 85748, Germany. yingbai.hu@tum.de.

${ }^{4}$ Yan Shi is with School of Automation Science and Electrical Engineering, Beihang University, 100083, Beijing, China. shiyan@buaa.edu. cn.
}

surgical robots was completed by Beretta et al. in [5]. A human-like reaching motion had been proposed in [6] with robot-environment interactions. A human-like path planning of the hand pose was achieved using a feed-forward Artificial Neural Network (ANN) in [7]. Nevertheless, these achievements only concentrated on the motion of end-effectors without considering the arm pose, which cannot achieve whole-body control [8] of mimicking human-like behavior on manipulators.

In order to solve the mentioned limitations, Kim et al. [9] presented the definition of the elbow swivel motion angle of the human arm, as shown in shown in Fig. 1. A wristelbow-in-line approach was implemented in [10] to project human-like kinematics resolution on the real robot by mapping the human demonstrated elbow angle. Zanchettin et al. [11] redefined the elbow swivel angle and mapped the modeled human-like swivel motion to Yumi robot to imitate human behavior. Moreover, the association between the hand pose and elbow swivel angle was investigated in [12]. A human-like delivery was attained using the nonlinear relation between the hand pose and the elbow swivel angle [11].

A single hidden layer feed-forward neural network (FFNN) was utilized in our previous work for mapping the hand pose to the swivel angle; thus imitating humanlike motion in real-time [13]. Moreover, the human-like performance was demonstrated in a teleoperated surgical scenario [14]. There are many works for data science, including the machine learning (ML) technique, big data and imitation learning using for the manipulator system. However, these

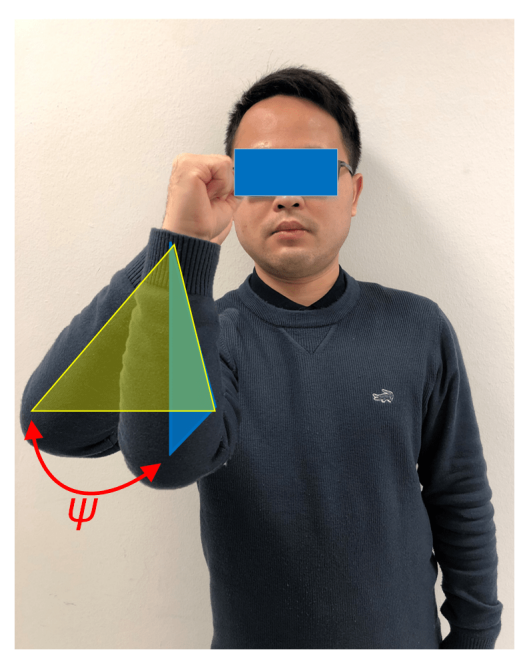

Fig. 1: Human elbow swivel motion angle. 


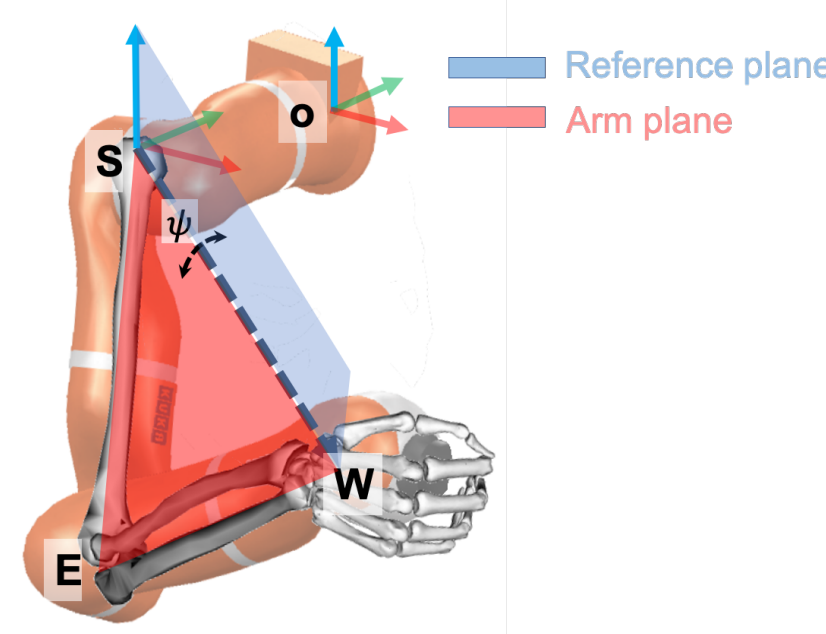

Fig. 2: Human-like kinematic mapping on the KUKA anthropomorphic manipulator.

processing of vast data set imposes a high computational burden in real-time system.

Low-speed computation and overfitting of neural networks (NN) models cannot meet the requirements for efficient learning in a dynamic circumstance. The big data processing techniques provide the possibility to make the robot imitating human-like behavior. They are widely applied on humanoid robots [15] (e.g., Atlas robots at Boston Dynamics [16]), evolution of computing (e.g., AlphaGo [17], Internet of Things (IoT) [18]), and human-robot interaction. Since the traditional continuous learning procedure is costly and timeconsuming, to design and develop a fast evolution method in the dynamic human-like learning procedure is necessary. We utilize machine learning based algorithms to model the human motion skill [19] and transfer the trained to model to achieve human-like motion on the robot manipulators. The Root Mean Square Error (RMSE) using the human motion data is utilized to validate the effectiveness of the implemented methods. The established model is then translated to manage whole-body human-like kinematic control of a manipulator [20].

In this paper, we propose an ML framework based on the deep convolutional neural network (DCNN) approach for fast online human-like motion learning and accuracy enhancement. The designed DCNN structure is to investigate the nonlinear relation between the human hand pose and the human swivel angle thus enhancing the quality of the regression analysis for a human-like movement model.

This paper is structured as follows: Section II introduces methodologies and system architecture. The regression performance between proposed algorithms and previous methods are compared, and the demonstration experiment using a KUKA anthropomorphic manipulator is presented in Section III. Section IV makes a summary and concludes further work.

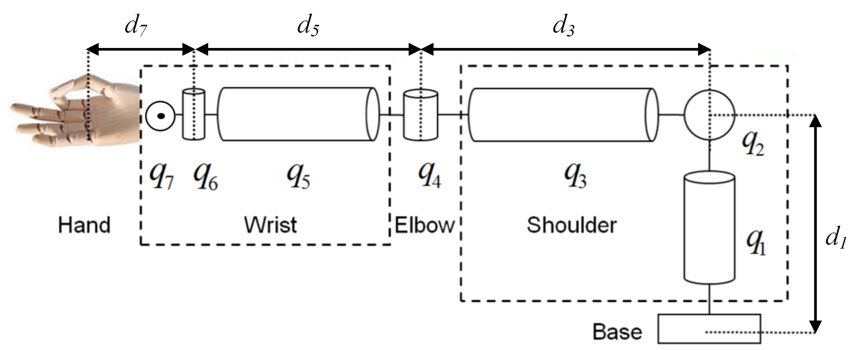

Fig. 3: The kinematic chain of a human upper limb. $q_{i}, i=$ $1, \cdots, 7$ and $d_{j}, j=1,3,5,7$ delegate the joint positions and the link lengths of each segment, respectively.

\section{Methodology}

Firstly, we present a kinematic model of the manipulator and then analyze the the swivel motion in manipulated tasks. A machine learning based model online training framework is then presented. Finally, the trained human-like motion model is transferred to the robot using a decoupled control approach.

\section{A. Human upper limb kinematic modeling}

To obtain the human-like motion model and achieve a human-like kinematic mapping on the robot (Fig. 2), the human arm is modeled as a 7 DoFs rigid kinematic chain as shown in Fig. 3. The coordination positions of the shoulder, the elbow, the wrist, and the hand are labeled as S, E, W, and $\mathrm{H}$, separately. $\psi$ denotes the elbow swivel angle between the desired way to arm. The expression of the elbow swivel angle $\psi$ has been presented in our previous works [13], [21]. As it has been discussed in the previous works [13], [21], the joint angles $\left(q_{i}, i=1,2, \cdots, 7\right)$ is calculated according to the geometry relation, and the hand pose is obtained based on the forward kinematics function using Denavit-Hartenberg (D-H) parameters [22]. Then, the angle between the reference plane and the arm plane can be adopted to describe the swivel angle $\psi$ [13].

\section{B. Deep learning based regression model}

In order to establish the relationship between the angle and hand pose, there are several approaches are adopted in our previous works [13], [23], [24], such as curve fitting (CF), feed-forward neural network (FFNN), and cascadeforward neural networks (CFNN). The complicated function structure of ANN-based methods often cause the problems of overfitting, underfitting, and time-consuming, even if they demonstrates a promising performance [13]. Hence, we proposed a novel regression framework based on deep convolutional neural network (DCNN) for fast computation and resolving the overfitting [21] by adding batch normalization, Rectified Linear Unit (ReLU) activation function, and dropout layer. Fig. 4 shows the DCNN structure in details, including two convolutional modules, dropout layer, and a regression layer. It is worth mentioning that the DCNN model can adopt multiple layers because it is safe from gradient vanishing problem. 


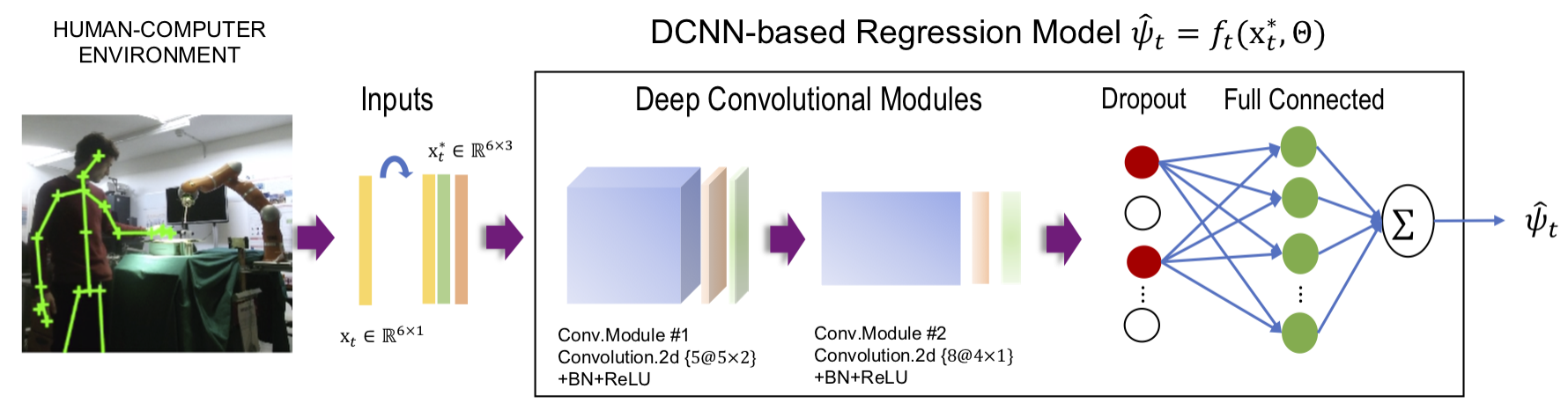

Fig. 4: The schematic diagram of DCNN-based framework for control of anthropomorphic manipulators. The obtained input $\mathbf{x}_{t}^{*}$ are processed by the DCNN regression model $\psi_{t}=f\left(\mathbf{x}_{t}^{*}, \Theta\right)$.

To reduce the online computational time and obtain high predictive accuracy, the DCNN model is designed with several optimization processing. Since convolutive layers can apply the same filter to different portions of the input data [25], they are good for pixels or sequences of the homogenous dataset. Hence, we extend the raw 6-D vector $\mathbf{x}$ into a homogenous matrix $\mathbf{x}^{*} \in \mathbb{R}^{6 \times 3}$ as:

$$
\mathbf{x}^{*}=\left[\mathbf{x} ; \mathbf{x}-\overline{\mathbf{x}} ; \frac{\mathbf{x}-\overline{\mathbf{x}}}{\sigma \mathbf{x}}\right]
$$

where $\sigma \mathbf{x}$ and $\overline{\mathbf{x}}$ are the standard deviation and average of $\mathbf{x}$, respectively. In the DCNN structure, the first convolutional module (Conv.Module \#1) consists of a 2-D convolutive layer with five kernels of size $2 \times 2$, a batch normalization (BN) with five channels and a ReLU activation function. The second convolutional module (Conv.Module \#2) takes the output from the first deep convolutional module as the inputs and filters them with eight kernels of size $2 \times 2$, followed by the BN and ReLU layers. The dropout layer (with 0.3 percentage) is used to reduce the training time. The fully connected layer vectorizes the previous outputs into 144 -dimensional features. The learning rate is 0.001 , and the corresponding optimization algorithm is used adaptive moment estimation optimizer (Adam). We set the minimum batch size as 50 samples, 0.001 drop factor, and 500 drop period. The DCNN-based online learning approach works well with continuous updating but is typically time-consuming with increasing of the testing dataset.

The supervised machine learning mechanism is used to validate the performance of the proposed framework. The built DCNN model $\hat{\psi}=f(\mathrm{x}, \Theta)$ on the training dataset will be used to predict the elbow swivel angle on testing dataset. The overall parameters $\Theta$ include the weights matrix and bias vectors of each layer. The purpose of nonlinear modeling is to fine the optimal parameters using the minimum least squares as follows.

$$
\begin{aligned}
\Theta & =\underset{\Theta}{\operatorname{argmin}} \sum_{t=1}^{N}\left(\hat{\psi}_{t}-\psi_{t}\right)^{2} \\
& =\underset{\Theta}{\operatorname{argmin}}\|\hat{\psi}-\psi\|_{2}^{2}
\end{aligned}
$$

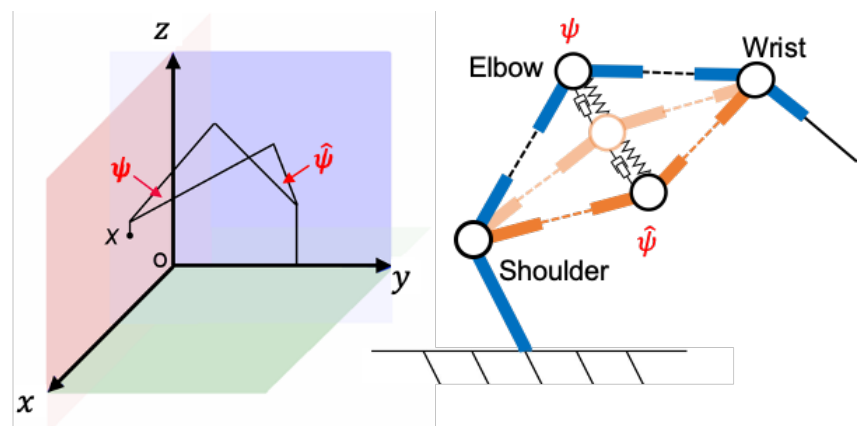

Fig. 5: Control of a 7 DoF robot manipulator with swivel motion.

The performance of DCNN model is evaluated by Root Mean Square Error (RMSE) at time $t$ as follows.

$$
\text { RMSE }=\sqrt{\sum_{t=1}^{N}\left(\frac{\hat{\psi}-\psi}{t}\right)^{2}}
$$

$N$ is the length of the whole dataset. A lower RMSE value is expected.

\section{Control of robot manipulator}

As it is shown in Fig. 5, given the desired Cartesian trajectory $X$, the elbow joint of the robot should be moved from the actual swivel angle $\psi$ to the human-like one $\hat{\psi}$ which can be obtained from the trained neural networks model. An extension of the analytical inverse kinematic method was carried out in our previous work [13]. However, the proposed method is not easy to transfer to other general robot manipulator. Hence, we achieve the human-like control with a decoupled control framework which is shown in Fig. 6. A main task projection [8] using Jacobean matrix to project the Cartesian velocity $\dot{X}$ to the joints velocity $\ddot{q}$ is introduced here. Then an impedance controller is adopted to generate the task joint torque $\tau_{T}$ to drive the manipulator. After getting the desired joint angles, the adaptive fuzzy control proposed in [26] is implemented to control the robot manipulator considering the external interaction force $F_{e}$ using an adaptive term $\tau_{F}$. Above all, the control scheme is integrated with two layers: an inner layer to guarantee the 


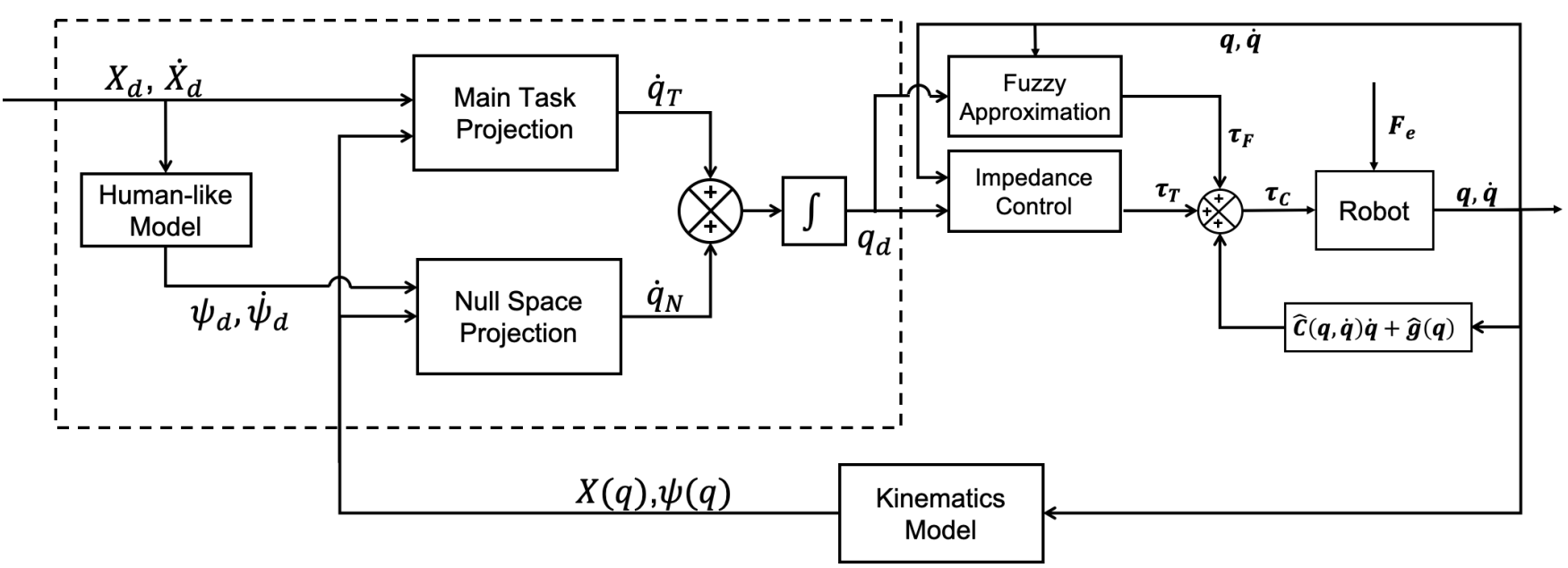

Fig. 6: The control diagram of human-like control scheme.

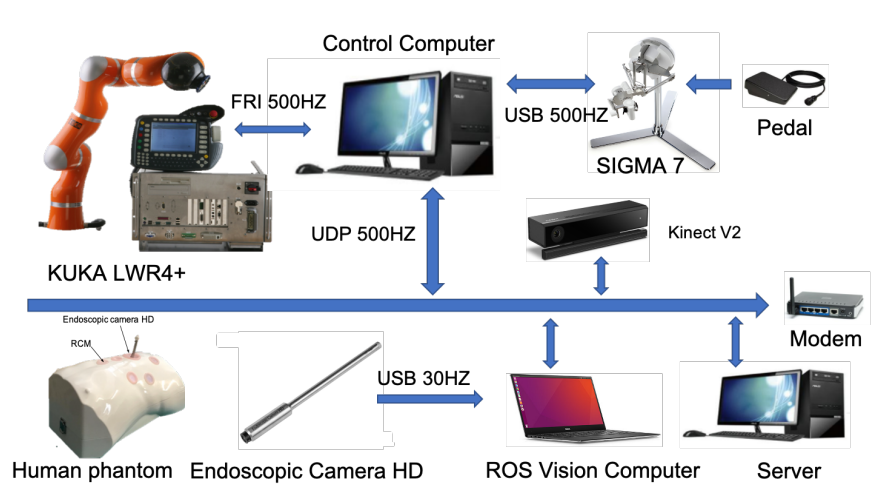

Fig. 7: The overview of the system setup.

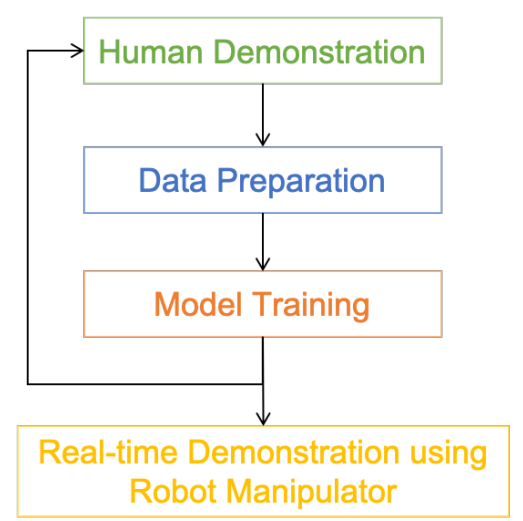

Fig. 8: Operation procedures.

control accuracy and an outer layer is introduced to achieve human-like manipulator control. The other corresponding detailed definition of control terms can be found in our previous works [8], [26].

\section{EXPERIMENT AND DEMONSTRATION}

\section{A. System Setup and Procedures}

The overview of the system setup is shown in Fig. 7. A KINECT V2 is used to collect the human motion data.

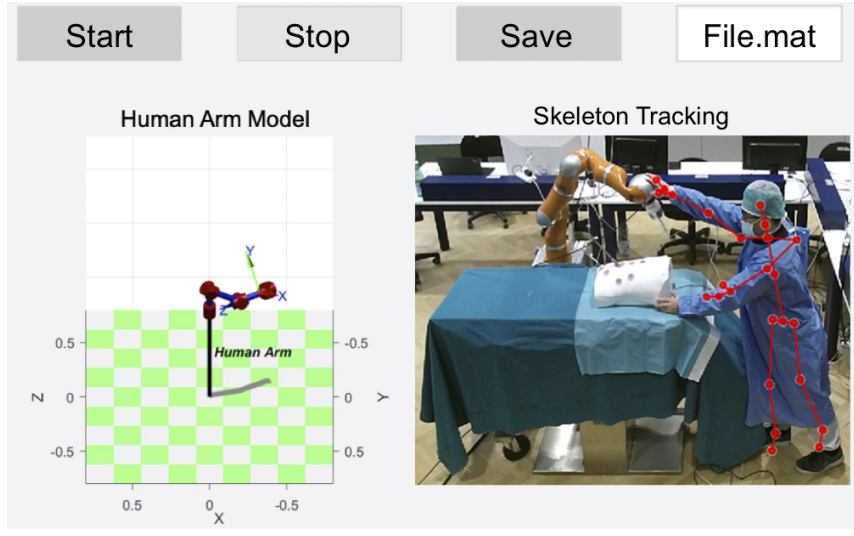

Fig. 9: The human-computer interface (HCI) for skeleton tracking.

The data is prepossessed on the server and is trained to model the human-like motion skill. Once the human skill model is trained, the trained model is applied to control the teleoperated KUKA manipulator. Sigma 7 is used as a master manipulator to move the robot manipulator via teleoperation. An endoscopic camera is placed in front of the user to enable the remote vision. The whole system has been developed using OROCOS ${ }^{1}$, with a real-time Xenomaipatched Linux kernel, and using ROS $^{2}$ Kinetic under Ubuntu. The operation procedures are shown in Fig. 8. Firstly, human demonstration are performed to collect the human motion data. Then data preparation using the human arm kinematic model to extract the required data for training the humanlike motion model. Finally, the trained model is applied on the real-time demonstration using robot manipulator.

\section{B. Data Preparation}

Fig. 9 shows the acquisition software interface for the skeleton tracking and swivel angle calculation by using the KINECT V2 (Microsoft, USA) [27] on Matlab 2018b

\footnotetext{
${ }^{1}$ Open Robotic Control Software, http://www.orocos.org/

${ }^{2}$ Robot Operating System, http://www.ros.org/
} 


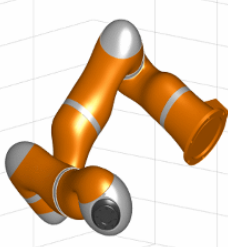

(1)

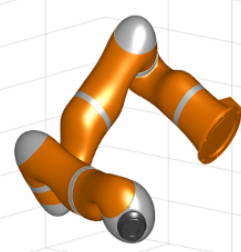

(2)

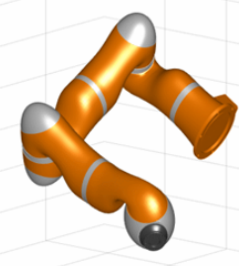

(3)

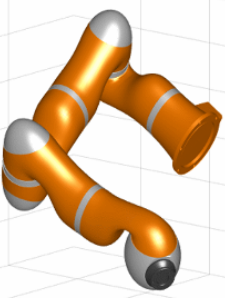

(4)

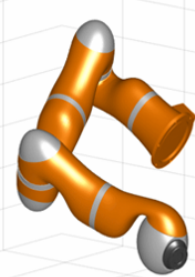

(5)

Fig. 10: Demonstration using robot simulator.
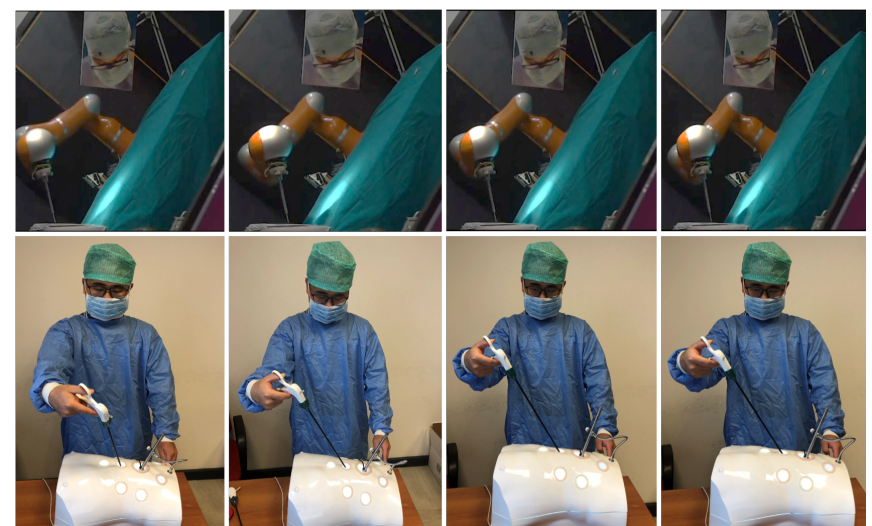

Fig. 11: Human-like motion demonstration on KUKA LWR4+.

platform. After activating the visual system at the upward side of the skeleton viewer by pushing the "start" button, the skeleton information of hand pose and swivel angle can be captured. The acquired data are saved for building the human motion model [13].

By adopting the proposed human-like kinematic model in sub-section II-A, the 6-D inputs $\left(\mathbf{x}_{t}=\left[x, y, z, \theta_{x}, \theta_{y}, \theta_{z}\right]_{t}\right)$ can be acquired from the 25 joints data. Meanwhile, the observed swivel angle $\psi_{t}$ is computed by the relationship of reference plane and arm plane. Finally, we collected five trajectory datasets to evaluate the performance of the proposed DCNN-based regression model in sub-section IIB. Each dataset have more than 2000 samples.

\section{Protocol}

To improve the evaluation accuracy, we use the leave-oneout strategy to train the DCNN model based four trajectory datasets and test on the last one. Furthermore, two ANNbased models (i.e., FFNN and CFNN) [28] and LSTM model are chosen for comparing with the DCNN model. According to our previous work [13], [23], the FFNN and CFNN models with single hidden layer and 20 neurons are the best structure for predicting the swivel angle. To validate the effectiveness of the proposed DCNN framework, we compute three types of performance, namely root mean squared error (RMSE), online prediction time $(\overline{c t})$ and the total time $\left(\sum c t\right)$ by quantitative evaluations. The experiments are implemented on 2.80-GHz Intel i5 CPU processor and 16.0 GB RAM with MATLAB 2018b platform.

\section{Performance comparisons of human motion skill model- ing}

All of the regression models are built on the four training datasets (about 6000 samples), and the performance of them are evaluated on the last trajectory groups (about 1500 samples). Table I shows the comparison RMSEs and cumulative predictive time $c t$ of DCNN, LSTM, FFNN, and CFNN. All of the results are averaged over ten runs. For a fair comparison, the LSTM structure is designed with a 150 neurons LSTM layer, a dropout layer with 0.3 percentage. We still use Adam as the estimation optimizer with 0.01 learning rate. The minimum batch size is 50 . The drop factor and period are 0.02 and 5 , respectively.

TABLE I: The comparative results of DCNN, LSTM, FFNN, and CFNN models on the four trajectories.

\begin{tabular}{cccc}
\hline Model & RMSE & $\overline{c t}$ & $\sum c t$ \\
\hline DCNN & $0.0791 \pm 0.0056$ & $0.08 \pm 0.006$ & $162.11 \pm 2.71$ \\
LSTM & $0.3114 \pm 0.0835$ & $0.71 \pm 0.069$ & $1328.21 \pm 9.16$ \\
FFNN & $0.1848 \pm 0.0677$ & $0.09 \pm 0.005$ & $198.70 \pm 3.40$ \\
CFNN & $0.1948 \pm 0.0913$ & $0.11 \pm 0.007$ & $204.81 \pm 3.22$ \\
\hline
\end{tabular}

As expected, the proposed DCNN framework shows good performance in terms of accuracy. It only needs around 162.11 seconds to predict all of the results in each trajectory, and 0.08 second to acquire a single result. Meanwhile, the DCNN model obtains the lowest RMSE among all the methods. Although ANN-based approaches can get a lower computational time, they obtain a higher RMSE values than DL-based methods.

\section{E. Demonstration of human-like redundancy optimization}

In this subsection, a human-like redundancy optimization combined with the trained model is used to illustrate the proposed method. The anthropomorphic robot manipulator pose is controlled via teleoperation and is computed by utilizing an interpolation algorithm. The robot pose are used as the inputs for the DCNN model for model training. Then 
the trained regression model is used to predict the humanlike swivel motion angle, and the decoupled control strategy is utilized to obtain the joints configuration. Finally, the demonstration experiments are performed with both simulation and real robot. As illustrated in Fig. 10 and Fig. 11, the robotic manipulator with the trained human-like motion model can imitate the human kinematics strategies when executing tracking tasks, and achieve human-like arm posture during manipulating tasks.

\section{CONCLUSiOn AND Future WORK}

This paper proposes a machine learning based on DCNN structure for the human motion skill transferring. This scheme can be implemented for human-like motion control of anthropomorphic manipulators. The experimental results show the proposed approach features with fast computation and accuracy enhancement in the online regression procedure and the trained human-like kinematic model can be utilized to manage the redundancy control of a 7 DoFs anthropomorphic robot manipulator. As a demonstration, the human-like control of the manipulator is achieved both on simulation and real robot. Furthermore, the proposed approach can also be employed to achieve human-like kinematic control on other similar anthropomorphic manipulators.

\section{REFERENCES}

[1] D. Wei, Z. Li, Q. Wei, H. Su, B. Song, W. He, and J. Li, "Humanin-the-loop control strategy of unilateral exoskeleton robots for gait rehabilitation," IEEE Transactions on Cognitive and Developmental Systems, 2019

[2] Z. Li, B. Huang, A. Ajoudani, C. Yang, C.-Y. Su, and A. Bicchi, "Asymmetric bimanual control of dual-arm exoskeletons for humancooperative manipulations," IEEE Transactions on Robotics, vol. 34, no. 1, pp. 264-271, 2017.

[3] Z. Li, J. Li, S. Zhao, Y. Yuan, Y. Kang, and C. P. Chen, "Adaptive neural control of a kinematically redundant exoskeleton robot using brain-machine interfaces," IEEE transactions on neural networks and learning systems, vol. 30, no. 12, pp. 3558-3571, 2018.

[4] Y. Liu, W. Su, Z. Li, G. Shi, X. Chu, Y. Kang, and W. Shang, "Motorimagery-based teleoperation of a dual-arm robot performing manipulation tasks," IEEE Transactions on Cognitive and Developmental Systems, vol. 11, no. 3, pp. 414-424, 2018.

[5] E. Beretta, E. De Momi, F. Rodriguez y Baena, and G. Ferrigno, "Adaptive hands-on control for reaching and targeting tasks in surgery," International Journal of Advanced Robotic Systems, vol. 12, no. 5 , p. 50, 2015.

[6] A. Atawnih, D. Papageorgiou, and Z. Doulgeri, "Reaching for redundant arms with human-like motion and compliance properties," Robotics and Autonomous Systems, vol. 62, no. 12, pp. 1731-1741, 2014.

[7] E. De Momi, L. Kranendonk, M. Valenti, N. Enayati, and G. Ferrigno, "A neural network-based approach for trajectory planning in robothuman handover tasks," Frontiers in Robotics and AI, vol. 3, p. 34, 2016.

[8] H. Su, C. Yang, G. Ferrigno, and E. De Momi, "Improved human-robot collaborative control of redundant robot for teleoperated minimally invasive surgery," IEEE Robotics and Automation Letters, vol. 4, no. 2, pp. 1447-1453, 2019.

[9] S. Kim, C. Kim, and J. H. Park, "Human-like arm motion generation for humanoid robots using motion capture database," in 2006 IEEE/RSJ International Conference on Intelligent Robots and Systems, pp. 3486-3491.

[10] W. Liu, D. Chen, and J. Steil, "Analytical inverse kinematics solver for anthropomorphic 7-dof redundant manipulators with human-like configuration constraints," Journal of Intelligent \& Robotic Systems, vol. 86, no. 1, pp. 63-79, 2017.
[11] A. M. Zanchettin, L. Bascetta, and P. Rocco, "Acceptability of robotic manipulators in shared working environments through human-like redundancy resolution," Applied ergonomics, vol. 44, no. 6, pp. 982989, 2013.

[12] C. Lamperti, A. M. Zanchettin, and P. Rocco, "A redundancy resolution method for an anthropomorphic dual-arm manipulator based on a musculoskeletal criterion," in 2015 IEEE/RSJ International Conference on Intelligent Robots and Systems, pp. 1846-1851.

[13] H. Su, N. Enayati, L. Vantadori, A. Spinoglio, G. Ferrigno, and E. De Momi, "Online human-like redundancy optimization for teleoperated anthropomorphic manipulators," International Journal of Advanced Robotic Systems, vol. 15, no. 6, p. 1729881418814695 , 2018.

[14] H. Su, C. Yang, H. Mdeihly, A. Rizzo, G. Ferrigno, and E. De Momi, "Neural network enhanced robot tool identification and calibration for bilateral teleoperation," IEEE Access, vol. 7, pp. 122 041-122 051, 2019.

[15] A. Goswami and P. Vadakkepat, Humanoid robotics: A reference. Springer, 2019.

[16] G. Nelson, A. Saunders, and R. Playter, "The petman and atlas robots at boston dynamics," Humanoid Robotics: A Reference, pp. 169-186, 2019.

[17] H. Su, S. Maji, E. Kalogerakis, and E. Learned-Miller, "Multiview convolutional neural networks for $3 \mathrm{~d}$ shape recognition," in Proceedings of the IEEE international conference on computer vision, 2015, pp. 945-953.

[18] M. Gusev and S. Dustdar, "Going back to the roots-the evolution of edge computing, an iot perspective," IEEE Internet Computing, vol. 22, no. 2, pp. 5-15, 2018.

[19] Z. Li, Y. Yuan, L. Luo, W. Su, K. Zhao, C. Xu, J. Huang, and M. Pi, "Hybrid brain/muscle signals powered wearable walking exoskeleton enhancing motor ability in climbing stairs activity," IEEE Transactions on Medical Robotics and Bionics, vol. 1, no. 4, pp. 218-227, 2019.

[20] X. Wu and Z. Li, "Cooperative manipulation of wearable dual-arm exoskeletons using force communication between partners," IEEE Transactions on Industrial Electronics, 2019.

[21] H. Su, W. Qi, C. Yang, A. Andrea, G. Ferrigno, and E. De Momi, "Deep neural network approach in human-like redundancy optimization for anthropomorphic manipulators," IEEE ACCESS, 2019.

[22] C. Gaz, F. Flacco, and A. De Luca, "Identifying the dynamic model used by the kuka lwr: A reverse engineering approach," in 2014 IEEE International Conference on Robotics and Automation, pp. 1386-1392.

[23] H. Su, J. Sandoval, M. Makhdoomi, G. Ferrigno, and E. De Momi, "Safety-enhanced human-robot interaction control of redundant robot for teleoperated minimally invasive surgery," in 2018 IEEE International Conference on Robotics and Automation, pp. 6611-6616.

[24] H. Su, J. Sandoval, P. Vieyres, G. Poisson, G. Ferrigno, and E. De Momi, "Safety-enhanced collaborative framework for teleoperated minimally invasive surgery using a 7-dof torque-controlled robot," International Journal of Control, Automation and Systems, vol. 16, no. 6, pp. 2915-2923, 2018.

[25] A. Krizhevsky, "One weird trick for parallelizing convolutional neural networks," arXiv preprint arXiv:1404.5997, 2014.

[26] Z. Li, C.-Y. Su, G. Li, and H. Su, "Fuzzy approximation-based adaptive backstepping control of an exoskeleton for human upper limbs," IEEE Transactions on Fuzzy Systems, vol. 23, no. 3, pp. 555566, 2014.

[27] O. Patsadu, C. Nukoolkit, and B. Watanapa, "Human gesture recognition using kinect camera," in 2012 Ninth International Conference on Computer Science and Software Engineering, pp. 28-32.

[28] S. Goyal and G. K. Goyal, "Cascade and feedforward backpropagation artificial neural networks models for prediction of sensory quality of instant coffee flavoured sterilized drink," Canadian Journal on Artificial Intelligence, Machine Learning and Pattern Recognition, vol. 2, no. 6, pp. 78-82, 2011. 